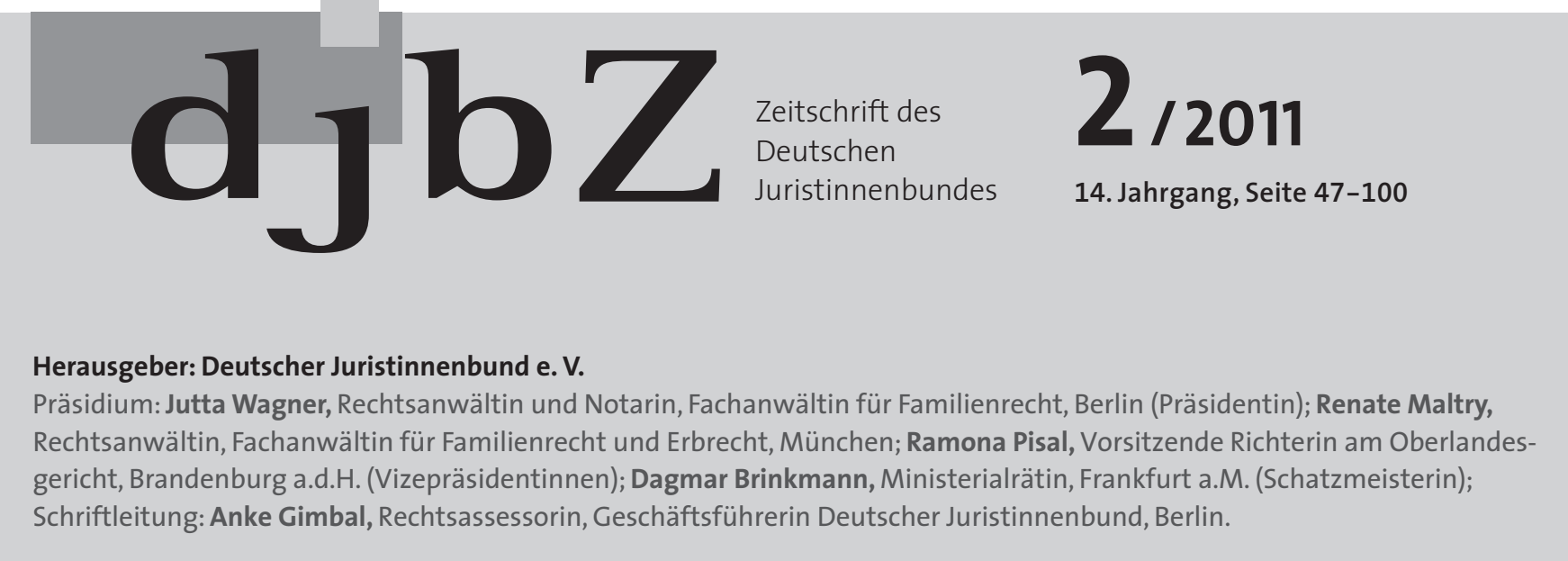

\title{
Geschlechtergerechte Pflege als Herausforderung für eine gleichstellungsorientierte Pflegepolitik
}

\author{
Heike Hoffer, LL.M. (Vanderbilt) \\ Mitglied der Kommission Recht der sozialen Sicherung, Famili- \\ enlastenausgleich des djb; Rechtsanwältin, Wissenschaftliche \\ Referentin im Arbeitsfeld „Alter, Pflege, Rehabilitation, Gesund- \\ heit" des Deutschen Vereins für öffentliche und private Fürsorge \\ e.V., Berlin
}

\section{Pflege ist weiblich}

„Pflege ist weiblich“. Diese Feststellung ist Grundlage und Ergebnis eines gleichstellungspolitisch geführten Pflegediskurses. Ausgehend von der Wissenschaft und gleichstellungspolitisch aktiven Organisationen wie dem djb ist dieser Diskurs mittlerweile im politischen Mainstream angekommen. „Pflege ist weiblich“ bedeutet, dass die Beteiligten an Pflegearrangements ${ }^{1}$ mehrheitlich Frauen sind: Weibliche Pflegebedürftige stellen in allen Altersgruppen jenseits des 75. Lebensjahrs die Mehrheit, bei den Altersgruppen ab dem 85. Lebensjahr sogar mehr als zwei Drittel der Pflegebedürftigen ${ }^{2}$. Auch professionell Pflegende (Pflegefachkräfte), insbesondere solche, die die direkte Pflege am Körper übernehmen, sind mit großer Mehrheit weiblich: Der Anteil der Frauen bei den Beschäftigten in den ambulanten Diensten liegt bei 88 Prozent; im stationären Bereich bei 85 Prozent. ${ }^{3}$ Informell, d.h. nicht beruflich und nicht professionell Pflegende (im SGB XI „Pflegepersonen“ genannt, zum Beispiel pflegende Angehörige, Freunde oder Nachbarn ${ }^{4}$ ) sind schließlich ebenfalls überwiegend Frauen. Hauptsächlich pflegen Töchter und Ehefrauen, gefolgt von den Schwiegertöchtern. ${ }^{5}$ Frauen pflegen im Alter von 45 bis 75 Jahren am häufigsten, während Männer erst in höheren Altersjahren pflegen, dann zumeist auch eher die Partnerin als Eltern oder Schwiegereltern. ${ }^{6}$ Pflegende insbesondere pflegende Frauen - sind zudem immer häufiger in einer sogenannten „Sandwichsituation“: Sie pflegen ältere
Verwandte, während die eigenen Kinder noch im Haushalt leben. ${ }^{7}$

\section{Ist Pflege geschlechtergerecht?}

Aus der Feststellung, dass Pflege weiblich ist, kann noch nicht per se geschlossen werden, dass Pflege nicht geschlechtergerecht organisiert ist. Hierfür bedarf es einer Bewertung, die auch die Analyse der Ursachen und Folgen des überproportional hohen Frauenanteils in der Pflege beinhaltet. Ob die „Weiblichkeit“ der Pflege zugleich Geschlechterungerechtigkeit bedeutet, soll hier durch einen Abgleich mit Kriterien, die

1 Mit dem Begriff des „Pflegearrangements“ wird hier das jeweilige Netzwerk aller beruflichen, freiwilligen und kommerziellen Unterstützungs- und Dienstleistungen bezeichnet, mit denen der (die) Pflegebedürftige seine (ihre) Versorgung sicherstellt (in Anlehnung an Barbara Mittnacht (2009), Qualitätsentwicklung und Nachhaltigkeit im Kontext häuslicher Pflegearrangements - Entwicklungstrends und Perspektiven, Dissertation: Bremen, S. 9).

2 Robert Koch Institut (2004): Schwerpunktbericht „Pflege” der Gesundheitsberichterstattung des Bundes, Berlin, S. 14. Als Pflegebedürftige sind hier nur die SGB XI-Leistungsbezieher erfasst.

3 Statistisches Bundesamt, Pflegestatistik 2009, Deutschlandergebnisse, S. 12, 21.

4 Vgl.zu den unterschiedlichen Erhebungsformen Rothgang, Heinz et al. (2009), GEK Pflegereport 2009, Schwäbisch-Gmünd, S. 14.

5 Schneekloth, Ulrich/Wahl, Hans Werner (Hrsg.) (2005): Möglichkeiten und Grenzen selbständiger Lebensführung in privaten Haushalten (MuG III), Integrierter Abschlussbericht, S. 41. Rothgang, Heinz et al. (2009), a.a.O., S. 14, stellen zudem fest, dass bei den „erheblichen“ Pflegetätigkeiten Männer nur zu einem Zehntel pflegen, bei den „geringeren“ Pflegetätigkeiten allerdings nahezu die Hälfte.

6 Schupp, Jürgen/Künemund, Harald (2004): Private Versorgung und Betreuung von Pflegebedürftigen in Deutschland, DIW Wochenbericht, 71, S. 289, 290.

7 Vgl. Backes, Gertrud et al. (2008): Geschlechterungleichheiten in der Pflege in: Bauer, Ullrich/Büscher, Andreas (Hrsg.): Soziale Ungleichheit und Pflege - Beiträge sozialwissenschaftlich orientierter Pflegeforschung, VS Verlag: Wiesbaden, S. 131, 132-133. 
umgekehrt für Geschlechtergleichheit bzw. Geschlechtergerechtigkeit stehen, geprüft werden. Dabei wird in diesem Beitrag in Anlehnung an Nancy Fraser ${ }^{8}$ Geschlechtergleichheit als ein Komplex von fünf Kriterien verstanden:

1. Bekämpfung der Armut/erhöhte eigene Absicherung;

2. Bekämpfung der Ausbeutung/Erhöhung der Machtressourcen von Frauen im Haushalt;

3. Gleichstellung in folgenden Bereichen: Gleiche Einkommen/Abbau Arbeitsmarktdiskriminierung, gleiche Freizeit/ Abbau diskriminierender Zeitstrukturen, gleiche Achtung/ Anerkennung der Arbeit von Frauen;

4. Bekämpfung der Marginalisierung/gleichberechtigte Teilhabe in allen Bereichen des gesellschaftlichen Lebens sowie

5. Bekämpfung des Androzentrismus/neue Normen für Frauen und Männer.

Ziel dieses Beitrags ist es, anhand der genannten Kriterien für Teilaspekte der Pflege zu prüfen, inwieweit Pflegepolitik geschlechtergerecht ist, und einige Hinweise dafür zu geben, wie sich gleichstellungspolitische Herausforderungen in der Pflege lösen lassen.

\section{Aktueller politischer Rahmen: Ist die Pflegepolitik der 17. Le- gislaturperiode auch Gleichstellungspolitik?}

Bundesminister Dr. Philip Rösler hat 2011 zum „Jahr der Pflege“ erklärt. ${ }^{9}$ Wird es auch ein Jahr der Gleichstellung? Zwei Kontrastpunkte:

Am 25. Januar 2011 wurde dem Bundesministerium für Familie, Senioren, Frauen und Jugend (BMFSFJ) das Gutachten der Sachverständigenkommission für den ersten Gleichstellungsbericht der Bundesregierung übergeben. ${ }^{10}$ Der Gleichstellungsbericht wurde auf Initiative des BMFSFJ erstellt und dient der Vorbereitung des zum Themenfeld „Gleichstellung“ im Koalitionsvertrag ${ }^{11}$ vereinbarten „Rahmenplans zur gleichberechtigten Teilhabe von Frauen und Männern in allen Phasen des Lebensverlaufs"12. In Bezug auf das Themenfeld private Pflege bzw. Sorgearbeit von Pflegenden fordert die Kommission beispielsweise die „Entwicklung einer konsistenten Lebenslaufpolitik (statt fragmentierter, an Effizienzkriterien orientierter Teilpolitiken), die einer weiteren Individualisierung der Risiken zum Beispiel von Phasen der Sorgearbeit entgegenwirkt“. ${ }^{13}$ Insbesondere sollte „sichergestellt werden, dass Unterbrechungen der Erwerbstätigkeit oder vorübergehende Verkürzungen der Arbeitszeit aufgrund von Sorgearbeit reversibel sind und nicht zu langfristigen Einkommensnachteilen führen“. ${ }^{14}$ Dabei sollten die „die Sorgearbeit berücksichtigenden Komponenten des Sozial- und Steuerrechts für die Pflege konsequent fortentwickelt und so ausgestaltet werden, dass die mit Einkommenseinbußen verbundenen Phasen der Sorgearbeit eine ausreichende soziale Absicherung (einschließlich einer Krankenversicherung während der Sorgetätigkeit) erfahren und dass eine eigenständige Alterssicherung im Lebensverlauf möglich bleibt." 15

Die Kommission weist auch darauf hin, dass „Erwerbsunterbrechungen und Arbeitszeitreduzierungen bei Kindererziehenden [...] deutlich besser abgesichert [sind] als solche von
Pflegenden." ${ }^{16}$ Hier empfiehlt die Kommission eine Angleichung durch eine Besserstellung der Pflegenden: „Vor dem Hintergrund der Auflösung der starren Altersstrukturierung und der unterschiedlichen Lebensverläufe von Männern und Frauen sollten dabei auch Pflegetätigkeiten jenseits des gesetzlichen Rentenalters noch zu einer Verbesserung der Rentenanwartschaften führen. Es ist zu evaluieren, inwieweit mit dem Gesetz über die Pflegezeit (PflegeZG) das Ziel der Vereinbarkeit von Beruf und familiärer Pflege erreicht wird. Hierzu sollten regelmäßig geschlechtsspezifische Daten, zum Beispiel zur Inanspruchnahme von Pflegezeit und hiermit verbundenen Problemen, erhoben werden. " 17 Die Kommission führt weiter aus: „Die Benachteiligung der Sorgearbeit für Pflegebedürftige im Vergleich zur Sorgearbeit für Kinder in der GRV ist nicht zu rechtfertigen. Eine bessere Anrechnung von Pflegezeiten auf die Rentenansprüche - auch für Nichterwerbstätige und Pflegende, die das Rentenalter bereits erreicht haben - ist im demografischen Wandel dringend geboten. Die Kommission empfiehlt daher, die rentenrechtliche Honorierung von Pflegearbeit zu verbessern [...]. Analoge Regelungen zur rentensteigernden Anrechnung von Kindererziehungs- und Pflegezeiten sind auch für solche Personengruppen zu schaffen, die nicht zu den abhängig Beschäftigten gehören. Die Anrechnung sollte - angelehnt an die additive Anrechnung von Kindererziehungszeiten - unabhängig von Erwerbsstatus und Alter der pflegenden Person sein. “18 Weitere Empfehlungen der Gutachterkommission werden an der jeweiligen Stelle in diesem Beitrag vorgestellt.

Am 23. März 2011 hat das Bundeskabinett den Gesetzentwurf über eine Familienpflegezeit beschlossen, der die Vereinbarkeit von Pflege und Erwerbsarbeit erleichtern soll. ${ }^{19}$ Das Vorhaben „Familienpflegezeit“ ist im Themenfeld „Pflege“ des Koalitionsvertrags vorgesehen. ${ }^{20}$ Auf den ersten Blick

8 Fraser, Nancy (1994): After the Family Wage. Gender Equity and the Welfare State. In: Political Theory, Vol. 22, No. 4, S. 591-618.

9 Siehe <http://www.bundesregierung.de/Content/DE/ Artikel/2010/12/2010-12-07-pflegegipfel.html> (Zugriff: 3.4.2011)

10 Neue Wege - Gleiche Chancen. Gleichstellung von Frauen und Männern im Lebensverlauf. Gutachten der Sachverständigenkommission für den ersten Gleichstellungsbericht der Bundesregierung, <http://www.fraunhofer.de/ueber-fraunhofer/geschaeftsstelle-gleichstellungsbericht /index.jsp> (Zugriff: 3.4.2011). Die Veröffentlichung durch die Bundesregierung und die Stellungnahme der Bundesregierung stehen bis dato (3.4.2011) noch aus.

11 Koalitionsvertrag für die 17. Legislaturperiode (2009-2013) der CDU, CSU und FDP vom 26.10.2009. Download unter: <http://www.cdu. de/doc/pdfc/og1026-koalitionsvertrag-cducsu-fdp.pdf> (Zugriff: 3.4.2011).

12 Vgl.zum „Rahmenplan“: Koalitionsvertrag, a.a.O., S. 73

13 Gutachten zum 1. Gleichstellungsbericht, a.a.O., S. 218.

14 Gutachten zum 1. Gleichstellungsbericht, a.a.O., S. 220.

15 Gutachten zum 1. Gleichstellungsbericht, a.a.O., S. 218.

16 Gutachten zum 1. Gleichstellungsbericht, a.a.O., S. 222.

17 Ebd.

18 Gutachten zum 1. Gleichstellungsbericht, a.a.O., S. 224

19 Vgl. statt aller: <http://www.faz.net/s/RuboEgEEF84AC1E4A389A8DC6C23161FE44/Doc EAD639E93BoD448C78B8DogAC AB346FFE ATpl Ecommon Scontent.html> (Zugriff: 3.4.2011). Der Gesetzentwurf liegt mittlerweile - in leicht veränderter Fassung als Regierungsentwurf dem Bundesrat als Drucksache 207-11 vom 15.4.2011 vor (Stand: 28.04.2011)

20 Koalitionsvertrag, a.a.O., S. 92. 
könnte vermutet werden, dass der Gesetzentwurf eine gleichstellungspolitische Maßnahme im Rahmen der Pflege ist. In der gleichstellungspolitischen Folgenabschätzung der Begründung zum Gesetzentwurf wird erfreut für den Gesetzentwurf ein „wertvoller gleichstellungspolitischer Beitrag“, der die Risiken unterbrochener Erwerbsbiografien von Frauen mindert und Anreize zu einer verstärkten Pflegetätigkeit von Männern schafft, konstatiert. ${ }^{21}$ Prüft man den Gesetzentwurf und die Begründung genauer, so wird jedoch schnell deutlich, dass dieser vorrangig dem Schutz von Arbeitgeber(inne)n und der Vermarktung von Versicherungsmodellen dient, aber kaum der Gleichstellung. ${ }^{22}$ Die Probleme des Entwurfs werden auch dadurch deutlich, dass in die Diskussionen die Empfehlungen der Gutachter(innen)kommission in dem vom gleichen Ministerium in Auftrag gegebenen Gutachten zum 1. Gleichstellungsbericht nicht einbezogen wurden und der Entwurf daher kaum Schritte in die von der Kommission geforderte Richtung unternimmt.

Der Koalitionsvertrag bietet aus gleichstellungspolitischer Sicht zwei weitere Kontrastpunkte: Bei Frauen wird die Erleichterung des Wiedereinstiegs ins Berufsleben - auch zum Beispiel nach einer pflegebedingten Unterbrechung der Erwerbstätigkeit - gefördert, nicht aber die Vermeidung des Ausstiegs aus dem Berufsleben. ${ }^{23}$ Für Jungen und Männer wird im Rahmen der Männerpolitik betont, dass diesen der Einstieg auch in pflegerische Berufe ermöglicht werden soll. ${ }^{24}$ Zwar ist ein Anstieg des Anteils männlicher professionell Pflegender grundsätzlich wünschenswert. Bisherige Erfahrungen haben aber gezeigt, dass selbst in der professionellen Pflege eine geschlechtsspezifische Differenzierung stattfindet: Frauen pflegen häufiger „direkt am Bett“, Männer tendieren stärker zu Verwaltungs- und Managementaufgaben. ${ }^{25}$ Allein die Erhöhung des Männeranteils in der Pflege wird daher nicht notwendig zu mehr Geschlechtergerechtigkeit in diesem Berufsfeld führen. Betrachtet man nunmehr das Verhältnis von professioneller und nicht professioneller Pflegearbeit, wird die Geschlechterungerechtigkeit noch deutlicher: Nach dem Verständnis der Koalitionäre sollen Frauen weiterhin unbezahlte Sorgearbeit leisten und sich mit der Möglichkeit eines verbesserten Wiedereinstiegs zufriedengeben, bei Männern wird vorrangig die bezahlte Pflegetätigkeit gefördert. Hier fehlt es mit Nancy Fraser - vor allem an der gleichen Anerkennung und Entlohnung von gleicher Arbeit - „Gleichstellung“ ist hierfür ein großes Wort.

Die nicht umgesetzten Koalitionsvereinbarungen zum Themenfeld „Pflege“ sollen in den Jahren 2011/2012 umgesetzt werden. Im Kern stehen die Fragen nach einer nachhaltigen Finanzierung der Pflegeversicherung und eines neuen, differenzierteren Pflegebedürftigkeitsbegriffs. Die Finanzierungsdiskussion wird gegenwärtig noch recht ergebnisoffen geführt: Sie mäandert zwischen den Vereinbarungen des Koalitionsvertrags, die neben dem bestehenden Umlageverfahren eine Ergänzung durch Kapitaldeckung vorsieht, die verpflichtend, individualisiert und generationengerecht ausgestaltet sein muss ${ }^{26}$, und den kürzlich öffentlich erhobenen Forderungen einiger Politiker(innen) nach (bloßer) Anhebung des Beitragssatzes um 0,5-0,6 Prozentpunkte. Diese und andere Vereinbarungen zum Themenfeld „Pflege“, unter anderem auch zur Weiterentwicklung der Pflegeausbildung, sind im Koalitionsvertrag nicht dezidiert in einen gleichstellungspolitischen Kontext gestellt worden. Man kann zusammenfassend feststellen: Pflegepolitik im Koalitionsvertrag befördert weder ausdrücklich noch in der bisherigen Umsetzung die Gleichstellung! ${ }^{27}$

\section{Gleichstellungspolitische Analyse der Pflege und Pflegepolitik}

Die gleichstellungspolitische Analyse soll hier anhand der drei an der Pflege und an Pflegearrangements hauptbeteiligten Gruppen erfolgen, in denen Frauen quantitativ die Mehrheit stellen: Pflegebedürftige, pflegende Angehörige und professionelle Pflegekräfte.

\section{Gleichstellungspolitische Analyse der Pflegepolitik für Pflege- bedürftige}

Pflegebedürftige sind von der Pflegepolitik in zwei zentralen Bereichen betroffen: Als Beitragszahler(innen) und als Leistungsempfänger(innen). ${ }^{28}$

\section{Frauen als Beitragszahlerinnen}

Als Beitragszahler(innen) scheinen bei einem bundeseinheitlichen, prozentual vom Einkommen abhängigen Beitragssatz für Frauen und Männer in der sozialen Pflegeversicherung zunächst keine Geschlechterungerechtigkeiten zu konstatieren zu sein. Der Beitragszuschlag von 0,25 Prozent für Kinderlose ( $\mathbb{S} 55$ Abs. 3 S. 1 SGB XI), der ebenfalls beide Geschlechter trifft, wird gleichstellungspolitisch häufig eher positiv bewertet. ${ }^{29}$ Lediglich die beitragsfreie Mitversicherung (Familienversicherung), die es auch in der Pflegeversicherung ( $\$ 25$ SGB $\mathrm{XI})$ gibt, begegnet - wie auch in der GKV - unter anderem aufgrund der damit verbundenen negativen Beschäftigungsanreize gleichstellungspolitischen Bedenken. Die Gutachterkommission für den 1. Gleichstellungsbericht fordert daher, „die

21 So die Begründung zum Referentenentwurf des Bundesministeriums für Familie, Senioren, Frauen und Jugend - Entwurf eines Gesetzes zur Vereinbarkeit von Familie und Beruf vom 24.2.2011, 18.00 Uhr, S. 21, wegen der hauptsächlich darin enthaltenen Regelung der Familienpflegezeit in Art. 1 des Gesetzes auch vereinfachend „Familienpflegezeitgesetz" (FamPflegeZG) genannt. Der mittlerweile vorliegende Regierungsentwurf vom 15.4.2011 übernimmt diese Begründung (S. 18).

22 Vgl. hierzu im Einzelnen die Stellungnahme des djb zum Regierungsentwurf für ein Familienpflegezeitgesetz.

23 Koalitionsvertrag, a.a.O., S. 73.

24 Koalitionsvertrag, a.a.O., S. 74

25 Backes, Gertrud et al. (2008), Gender in der Pflege - Herausforderungen für die Politik, Expertise im Auftrag der Friedrich-Ebert-Stiftung, Bonn, WISO Diskurs, S. 6.

26 Koalitionsvertrag, a.a.O., S. 93.

27 Auf die umfassende gleichstellungspolitische Analyse des Koalitionsvertrags von Margarete Schuler-Harms in djbZ 2010, 51 ff. wird ergänzend verwiesen.

28 In diesem Beitrag wird nur auf die Situation gesetzlich versicherter Pflegebedürftiger eingegangen.

29 Vgl. z.B. die Bewertung von Anne Lenze (2008) im Vortrag „Schluss mit der Familienförderung" beim Deutschen Familienverband, <http://www.deutscher-familienverband.de/index. php?id=3718\&no_cache=1\&sword_list[]=lenze > (Zugriff: 6.4.2011). 
beitragsfreie Ehegattenmitversicherung in der gesetzlichen Krankenversicherung [...] durch eine eigenständige soziale Sicherung [zu ersetzen], wobei für definierte Phasen der Sorgearbeit - ähnlich wie bei den Kindererziehungszeiten in der Rentenversicherung - die Gemeinschaft einzustehen hätte. “30

Das Verhältnis von Aufwendungen (Beiträgen) für die Pflegeversicherung zu Leistungen scheint auf den ersten Blick für Frauen positiv auszufallen: Frauen haben als Pflegebedürftige zwar im Durchschnitt kürzere Pflegedauern (bezogen auf Pflegebedürftigkeit im Sinnes des SGB XI ${ }^{31}$, die überlebenden Frauen jedoch einen durchschnittlich längeren Leistungsbezug in der häuslichen Versorgung aus der gesetzlichen Pflegeversicherung. ${ }^{32}$ In der stationären Versorgung erhalten Frauen den Löwenanteil der Ausgaben, da sie häufiger als Männer stationär versorgt werden. ${ }^{33}$ Frauen erhalten also über einen längeren Zeitraum und teurere Leistungen für den gleichen prozentualen Finanzierungsaufwand aus der Pflegeversicherung. Als Beitragszahlerinnen stehen sie also besser da als Männer oder?

Eine solche Betrachtungsweise blendet den Grund für den durchschnittlich kürzeren Leistungsbezug von Männern aus: Männer nehmen häufig erst dann Leistungen der Pflegeversicherung in Anspruch, nachdem sie über eine lange Zeit von ihren Partner(inne)n oder Töchtern/Schwiegertöchtern gepflegt wurden. ${ }^{34}$ Dadurch kommt es bei Männern zu einer späteren Beantragung von Pflegeleistungen und demzufolge einer geringeren Inanspruchnahme der Pflegeleistungen. ${ }^{35}$ Dies funktioniert für Frauen nicht in der gleichen Art und Weise: Zum einen sind viele Frauen im Alter alleinstehend, nicht selten weil der (zuvor von ihnen gepflegte) Partner mittlerweile verstorben ist, und daher auf professionelle Leistungen bereits früher im Pflegeverlauf und in größerem Maße angewiesen als Männer. Zum anderen kann davon ausgegangen werden, dass bei Frauen, die selbst jahrelang gepflegt haben, die physischen und psychischen Belastungen durch die Pflege (ihrer Partner oder Eltern/Schwiegereltern) dazu beitragen, ihr eigenes Risiko, pflegebedürftig zu werden, zu erhöhen. ${ }^{36}$ Schließlich werden Frauen - in Ermangelung eines pflegenden Partners - häufiger als Männer in relativ teureren vollstationären Pflegeeinrichtungen gepflegt, wodurch sich die Gesamtausgaben für die Frauen weiter erhöhen. ${ }^{37}$

Erstrebenswert ist für Frauen jedoch nicht, besonders viele Leistungen der Pflegeversicherung für ihren Beitrag zu erhalten. Erstrebenswert ist vielmehr, das Pflegerisiko von Frauen auf das mögliche Mindestmaß zu reduzieren. Daher sind aus gleichstellungspolitischer Sicht solche Maßnahmen sinnvoll, die das Pflegerisiko von Frauen allgemein, und auch das Pflegerisiko, das durch frauenspezifische Pflege- und andere Sorgetätigkeiten entsteht, zu verringern. Soweit eigene Pflegebedürftigkeit das Ergebnis von geleisteter Pflege- und Sorgetätigkeit ist, kann dies einerseits dadurch geschehen, dass der Männeranteil unter den Pflegenden erhöht wird und damit die Belastungen durch Pflege- und Sorgetätigkeiten nicht mehr überwiegend Frauen treffen. Andererseits kommen spe- zielle Gesundheitsförderungs- und Schutzmaßnahmen für Pflegende den betroffenen Frauen gegenwärtig in besonderem Maße zugute. Soweit ein erhöhtes Pflegerisiko von Frauen die Folge von lebenslaufbezogener Geschlechterungerechtigkeit ist, ist diese zu bekämpfen. Soweit es zum Beispiel in der Hochaltrigkeit von Frauen begründet liegt, ist die längere Lebenserwartung, die dieser zugrundeliegt, natürlich per se positiv zu bewerten (hier haben eher die Männer einen Aufholund entsprechenden Unterstützungsbedarf). Geschlechtsspezifische Ungerechtigkeiten entstehen erst dort, wo das erhöhte Pflegerisiko im Alter aus geschlechtsspezifisch ungleichen Belastungssituationen im Lebensverlauf resultiert. ${ }^{38}$ Betrachtet man die Erkenntnisse der Gesundheitswissenschaften über die Ursachen von Krankheit und Pflegebedürftigkeit (z.B. die Risikoerhöhung durch Armut, mangelnde soziale Teilhabe, erhöhte physische und psychische Belastung, niedrige Wertschätzung), so kann festgehalten werden, dass die Umsetzung der in den Kriterien von Nancy Fraser enthaltenen Forderungen im Lebensverlauf von Frauen dazu beitragen wird, Krankheitsrisiken und das Pflegerisiko von Frauen insgesamt zu senken.

Soweit Frauen umfangreich Pflegeleistungen erbracht und die Pflegeversicherung aufgrund des dadurch verringerten Leistungsbezugs von Männern entlastet haben, könnte zudem überlegt werden, diese Pflegeleistung auch durch eine Ermäßigung des Beitragssatzes zu honorieren. Das BVerfG hat die Verfassungsmäßigkeit des oben beschriebenen Beitragszuschlags für Kinderlose in der Pflegeversicherung damit begründet, dass durch die private Erziehungsleistung „Versicherten ohne Kinder im Versicherungsfall ein Vorteil aus der Erziehungsleistung anderer beitragspflichtiger Versicherter [erwächst], die wegen der Erziehung zu ihrem Nachteil auf Konsum und Vermögensbildung verzichten". 39 Das BVerfG führt weiter aus: „Wenn aber ein soziales Leistungssystem ein Risiko abdecken soll, das vor allem die Altengeneration trifft, und seine Finanzierung so gestaltet ist, dass sie im Wesentlichen nur durch das Vorhandensein nachwachsender Generationen funktioniert, die jeweils im erwerbsfähigen Alter als

30 Gutachten zum 1. Gleichstellungsbericht, a.a.O., S. 218.

31 Müller, Rolf et al. (2010): Reicht eine zweijährige Familienpflegezeit für Arbeitnehmer? In: Soziale Sicherheit 6-7 (10), S. 230 ff., gehen aufgrund von Neuberechnungen von durchschnittlichen Pflegedauern in der häuslichen Pflege von ca. 3 (Frauen) bis 4 (Männer) Jahren aus.

32 Rothgang, Heinz et al. (2010), Barmer GEK Pflegereport 2010, S. 142.

33 Vgl. 4. Bericht über die Entwicklung der Pflegeversicherung (2010), BT-Drucks. 16/7772 v. 17.1.2008, S. 26, <http://dipbt.bundestag.de/ dip21/btd/16/077/1607772.pdf> (Zugriff: 10.4.2011).

34 Statistisches Bundesamt, Pflegestatistik 2009, Deutschlandergebnisse, S. 7 .

35 Ebd.

36 Robert Koch Institut (2004), a.a.O., S. 15.

37 Statistisches Bundesamt, Pflegestatistik 2009, Deutschlandergebnisse, S. 8.

38 Eine solche lebenslaufbezogene Analyse geschlechtsspezifischer Ursachen des Pflegerisikos im Alter ist aufgrund der Datenlagen bislang schwierig durchzuführen. Sie würde auch den Rahmen dieses Beitrags sprengen. Daher soll hier auf die Notwendigkeit einer solchen Analyse nur hingewiesen werden.

39 BVerfG, Urteil vom 3.4.2001 - 1 BvR 1629/94, NJW 2001, S. 1712, 1714. 
Beitragszahler die mit den Versicherungsfällen der vorangegangenen Generationen entstehenden Kosten mittragen, dann ist für ein solches System nicht nur der Versicherungsbeitrag, sondern auch die Kindererziehungsleistung konstitutiv. Wird dieser generative Beitrag nicht mehr in der Regel von allen Versicherten erbracht, führt dies zu einer spezifischen Belastung kindererziehender Versicherter im Pflegeversicherungssystem, deren benachteiligende Wirkung auch innerhalb dieses Systems auszugleichen ist. Die kindererziehenden Versicherten sichern die Funktionsfähigkeit der Pflegeversicherung also nicht nur durch Beitragszahlung, sondern auch durch Betreuung und Erziehung von Kindern“. ${ }^{40}$

Angelehnt an diese Argumentation wäre zu überlegen, welcher „generative“ oder sonstige „Beitrag“ durch die Pflegeleistung von informell Pflegenden zugunsten der „Funktionsfähigkeit der Pflegeversicherung “ erbracht wird. Denn informell Pflegende entlasten die Pflegeversicherung in erheblichem Maße von Ausgaben für professionelle Leistungen. Würde die Pflege demgegenüber - wie es beispielsweise in Schweden oder Japan der Fall ist - in größerem Maße über professionelle Dienstleistungen gesichert, wäre dies unmittelbar beitragssatzrelevant und würde einen Beitragssatz erfordern, der nach Schätzungen von Expert(inn)en über dem Doppelten des gegenwärtigen Beitragssatzes läge. Die Pflegeleistung informell Pflegender ist zudem von unmittelbarerer Wirkung auf die Pflegeversicherung als die Erziehungsleistung von Eltern. Man könnte - in Anlehnung an die o.g. Formulierung des BVerfG - gar sagen: Versicherten, die keine Pflegebedürftigen informell unterstützten, erwächst ein Vorteil aus der Pflegeleistung anderer beitragspflichtiger Versicherter, die wegen der Pflege zu ihrem Nachteil auf Konsum und Vermögensbildung, Freizeit und soziale Teilhabe verzichten und stattdessen erhebliche Nachteile im Erwerbsleben, bei der Alterssicherung sowie erhöhte gesundheitliche Belastungen auf sich nehmen. ${ }^{41}$

Es könnte daher überlegt werden, bereits auf Beitragsseite den besonderen Beitrag von Pflegenden (unabhängig vom Geschlecht, aber derzeit überwiegend Frauen betreffend) zu würdigen. $\mathrm{Ob}$ eine weitere Ausdifferenzierung der Beiträge allerdings dem Solidaritätsgrundsatz des deutschen Sozialversicherungssystems entspricht und einer verfassungsrechtlichen Prüfung standhält, ist schon fraglich. Zudem könnte eine weitere Differenzierung immer weitere Begehrlichkeiten wecken und insgesamt den Grundsatz der Solidarität aushöhlen. Auch wäre jedenfalls bei einer Absenkung der Beiträge für Pflegende zu bedenken, dass dies der Pflegeversicherung auch Finanzmittel entzieht und zu einer Beitragssatzerhöhung insgesamt führen könnte. Schließlich - und dies scheint jedenfalls aus gleichstellungspolitischer Sicht das gewichtigste Argument ist fraglich, ob ein geringer prozentualer Abschlag für Pflegende überhaupt eine deutliche Verbesserung ihrer Lebenssituation bedeutet. Jedenfalls dürfte dies nicht dazu führen, dass mit einer „kompensatorischen“ Beitragssatzabsenkung für Pflegende der Eindruck entsteht, für Pflegende wäre damit alles getan, was erforderlich ist.

\section{Frauen als Pflegebedürftige}

Weiterhin muss die zweite Art der Betroffenheit Pflegebedürftiger berücksichtigt werden: die Leistungsseite. Wie $\mathbb{S} 3$ SGB XI feststellt, soll die Pflegeversicherung mit ihren Leistungen vorrangig die häusliche Pflege und die Pflegebereitschaft der Angehörigen und Nachbarn unterstützen (sog. „Vorrang der häuslichen Pflege“); die Leistungen der Pflegeversicherung „ergänzen“ insoweit die familiäre, nachbarschaftliche oder sonstige ehrenamtliche Pflege oder Betreuung ( $\mathbb{} 4$ Abs. 2 S. 1 SGB XI). Mit dieser Ergänzungsfunktion wird auch der sogenannte „Teilkaskocharakter" der Pflegeleistungen begründet, nämlich die Tatsache, dass die Pflegeleistungen - anders als die Leistungen der GKV - der Höhe nach gedeckelt sind und nicht die medizinisch (bzw. pflegerisch) notwendigen Pflegeleistungen abdeckt, sondern nur der Höhe nach begrenzte „Zuschüsse“ zur Unterstützung der Pflege leistet.

Dieser Teilkaskocharakter der Pflegeversicherung trifft Frauen überproportional. Denn von der umfassenden Absicherung der GKV profitieren Männer stärker als Frauen. ${ }^{42}$ Es kann vermutet werden, dass dies historisch auch darin begründet liegt, dass die GKV ursprünglich die Versicherung der - zu Entstehungszeiten wie heute - überwiegend männlichen Arbeitnehmer war, deren Arbeitskraft erhalten werden sollte, und Frauen überwiegend (nach Einführung der Familienversicherung) nur „akzessorisch“ versichert waren. Auf den Punkt gebracht bedeutet dies: Die gesellschaftliche Bewertung von männlich dominierter Erwerbsarbeit ist deutlich höher als die gesellschaftliche Bewertung des Autonomieerhalts und würdigen Alterns der dann die Mehrheit stellenden Frauen. Für Letzteres reicht „Teilkasko“.

Zwar waren bei Einführung der Pflegeversicherung die Beträge der Sachleistungen (z.B. $\$ 36$ SGB XI, $\mathbb{4} 43$ SGB XI) immerhin noch überwiegend bedarfsdeckend, jedenfalls soweit man nur die im SGB XI leistungsberechtigten Pflegebedürftigen und die vom SGB XI finanzierten Pflege- und Versorgungsleistungen betrachtet. In Ermangelung einer umfassenden Dynamisierung (diese erfolgte erstmalig 2008 mit dem Pflegeweiterentwicklungsgesetz) und angesichts der Tatsache, dass es nicht wenige Fälle von Pflegebedürftigkeit jenseits der Definition des SGB XI gibt ${ }^{43}$, sind die Pflege(sach)leistungen des SGB XI jedoch häufig nicht bedarfsdeckend. Zudem sind alle Kosten für Unterkunft und Verpflegung im Pflegeheim grundsätzlich nicht von den Leistungen der Pflegeversicherung umfasst, ebenso wenig wie - jedenfalls in der häuslichen Versorgung - Leistungen zur sozialen Teilhabe Pflegebedürftiger. Die mangelnde Bedarfsdeckung der Pflegeversicherung zeigt sich nicht zuletzt in dem Wiederanstieg der ergänzenden

40 BVerfG, Urteil vom 3.4.2001 - 1 BvR 1629/94, NJW 2001, S. 1712, 1715. 41 Zu den Leistungen an Pflegende im Einzelnen, insbesondere nach \$ $44 \mathrm{ff}$. SGB XI, vgl. unten „Frauen als Pflegende“.

42 König, Christina/Zoike, Erika (2004): Krankheitskosten nach Geschlecht und Alter - Sind Frauen wirklich teurer? In: Die BKK, S. $445 \mathrm{ff}$.

43 Also z.B. unterhalb der Erheblichkeitsschwelle des $\S 14$ Abs. 1 bzw. $\S 15$ Abs. 1 Ziff. 1 SGB XI, oberhalb der Pflegestufe III bzw. des Härtefalls oder für andere Pflegeleistungen als die in $\S 14$ Abs. 4 SGB XI genannten, also z.B. für die allgemeine Beaufsichtigung von Demenzkranken. 
Leistungen der Hilfe zur Pflege, die durch die Einführung der Pflegeversicherung zunächst stark entlastet wurde, seit einigen Jahren jedoch wieder erheblich steigende Ausgaben verzeichnet.

Diese unvollkommene Absicherung durch die Pflegeversicherung betrifft nun Frauen in besonderer Weise: Zunächst beschreibt $\mathbb{1} 1$ Absatz 1 SGB XI als Zweck der Einrichtung der sozialen Pflegeversicherung die „soziale Absicherung des Risikos der Pflegebedürftigkeit“. Gleichzeitig wird diese Absicherung durch den Teilkaskocharakter dezidiert begrenzt. Von dieser Leistungsbegrenzung sind Frauen mehrfach betroffen: Zunächst - im Falle der Pflegebedürftigkeit eines Partners durch ergänzend notwendige Selbstzahlungen des Partners, die häufig aus dem Gesamtfamilieneinkommen bestritten werden dürften oder jedenfalls das verfügbare Familieneinkommen schmälern. Weiterhin durch mögliche eigene Pflegetätigkeit, gegebenenfalls verbunden mit einem Verzicht oder einer Einschränkung der eigenen Erwerbstätigkeit (mit entsprechenden Folgen für weitere Erwerbsmöglichkeiten, berufliche Fortentwicklung und Alterssicherung). Dann - wenn der Partner verstorben ist - durch einen schlechteren eigenen Gesundheitszustand aufgrund der Pflegetätigkeit sowie gegebenenfalls den Verlust sozialer Kontakte aufgrund der Pflegezeit. Und schließlich - bei Eintritt einer eigenen Pflegebedürftigkeit - weitere ergänzend notwendige Zahlungen aus dem eigenen Einkommen, die insbesondere bei einer in Abwesenheit eines pflegenden Partners und aufgrund des jetzt höheren Lebensalters erhöhten Wahrscheinlichkeit, statt häuslicher Versorgung im Pflegeheim versorgt zu werden, eine erhebliche Höhe erreichen können und häufig mindestens mittelfristig ergänzende Sozialhilfeleistungen der Hilfe zur Pflege ( $\mathbb{S} \$ 61 \mathrm{ff}$. SGB XII) erforderlich machen kann (besonders dann, wenn die Alterseinkünfte aufgrund niedrigerer Löhne und sorgebedingter Auszeiten erheblich niedriger sind als bei einem Mann).

Mit anderen Worten: „Erst versorgen Frauen andere, dann werden sie selbst nicht angemessen versorgt. “44 Betrachtet man Nancy Frasers Kriterium „Vermeidung von Armut/Erhöhung der eigenen Absicherung“, so kann festgestellt werden, dass Frauen als Pflegebedürftige gegenüber Männern in erhöhtem Maße armutsgefährdet und durch den Teilkaskocharakter der Pflegeversicherung auch noch typischerweise schlechter abgesichert sind. Denn sie können zwar Pflegeversicherungsleistungen beanspruchen, von den in der Pflegeversicherung vorausgesetzten und zur Begründung ihres Teilleistungscharakters dienenden privaten Pflegeleistungen profitieren sie jedoch nicht in gleicher Weise. Dies wird nachfolgend kurz illustriert:

Frauen als mehrheitlich Pflegebedürftige werden häufiger in Pflegeheimen versorgt als Männer. ${ }^{45}$ Dies hat überwiegend mit der häufig fehlenden Unterstützungsmöglichkeit durch (bereits verstorbene) Partner, aber auch mit der längeren Lebenserwartung von Frauen und dem damit verbundenen Anstieg des Pflegerisikos zu tun. Befragungen zeigen, dass auch bei Frauen im höheren Lebensalter - wie bei allen Pflegebedürftigen - mehrheitlich der Wunsch nach einer Versorgung in der eigenen
Häuslichkeit besteht. Es wird deutlich, dass das in der Pflegeversicherung ( $\$ 2$ Abs. 2 SGB XI) und (eingeschränkt) im Sozialhilferecht ( $\ 9$ Abs. 2 SGB XII) verankerte Wunsch- und Wahlrecht der Pflegebedürftigen bezüglich der konkreten Ausgestaltung der Hilfen für Frauen faktisch beschränkter ist als für Männer. Zudem ist Heimpflege deutlich teurer. Häufig zehrt sie verbliebenes Einkommen und Vermögen im Zeitverlauf auf, so dass die betroffenen Frauen verstärkt auf ergänzende Sozialhilfeleistungen insbesondere zur Sicherung des Lebensunterhalts in Einrichtungen (d.h. Wohn- und Verpflegungskosten) angewiesen sind ( $\$ 35$ Abs. 1 SGB XII). Finanziell verbleibt ihnen in dieser Situation nur der Barbetrag des $\$ 35$ Absatz 2 SGB XII (27 \% des Eckregelsatzes, aktuell 98,28 Euro pro Monat) zur persönlichen Verfügung. Zudem ist der Empfang von Leistungen der Sozialhilfe nach wie vor mit einem gesellschaftlichen Stigma belegt. Dazu kommt, dass die Sozialhilfebedürftigkeit aufgrund eines Pflegeheimaufenthalts die Möglichkeit nimmt, in nennenswertem Umfang Vermögen zu vererben und der Vermögensaufbau und die finanzielle Stabilität von Familien über Generationen gefährdet sein können.

Um auch älteren Frauen den vorrangigen Verbleib in der eigenen Häuslichkeit zu sichern, ist die Unterstützung und Qualität von häuslichen Pflegearrangements sowie ein Leistungsrecht, das individuelle, flexible Arrangements überhaupt ermöglicht, von großer Bedeutung. ${ }^{46}$ Hier hat das Pflege-Weiterentwicklungsgesetz zwar einige Verbesserungen eingeführt: So wurde das sogenannte „Poolen von Leistungen“ möglich, bei dem mehrere Pflegebedürftige den gleichen ambulanten Pflegedienst in Anspruch nehmen und dadurch Effizienzgewinne haben sollen, mit denen weitergehende Betreuungsleistungen möglich werden. Auch wurde die sogenannte Kombinationsleistung, die die gleichzeitige, jeweils anteilige Inanspruchnahme von ambulanten Pflegedienst- und Pflegegeldleistungen ermöglicht, bei einer Kombination mit teilstationären Leistungen (d.h. Tagespflege und/oder Nachtpflege) auf 150 Prozent des bisherigen Gesamtwerts aufgestockt.

Beide - gesetzgeberisch grundsätzlich sinnvollen - Verbesserungen haben jedoch nach Berichten aus der Praxis bisher wenig praktische Auswirkungen: Das Poolen von Leistungen funktioniert danach nicht, weil ambulante Dienste bereits jetzt unterfinanziert sind und durch die Effizienzgewinne (z.B. die nur einmalige Anfahrt) nur das eigentlich notwendige Leistungsniveau finanziert wird, nicht aber ein echtes „Plus“ an ansonsten von der ambulanten Sachleistung nicht erfassten Betreuungsleistungen möglich wird. Die erhöhte Kombinationsleistung bei Inanspruchnahme teilstationärer Einrichtungen setzt voraus, dass es ein hinreichendes Angebot an qualitätsgesicherten teilstationären Einrichtungen gibt, was gegenwärtig nicht der Fall ist.

44 Formulierung in Anlehnung an einen mündlichen Kommentar von Prof. Dr. Kirsten Scheiwe, Universität Hildesheim.

45 Statistisches Bundesamt, Pflegestatistik 2009, Deutschlandergebnisse, S. 8.

46 Vgl. hierzu auch den Beitrag von Katja Nebe in diesem Heft. 
Der den Pflegekassen obliegende Sicherstellungsauftrag ( $\mathbb{6} 69$ SGB XI: insbesondere Sicherstellung einer bedarfsgerechten und gleichmäßigen Versorgung) bietet kaum Abhilfe: Denn in der Pflege gibt es keine Bedarfsplanung, und auch sonst haben die Pflegekassen wenig tatsächliche Einflussmöglichkeiten, wenn die Zahl oder regionale Verteilung von ambulanten Diensten und teilstationären Einrichtungen nicht dem eigentlichen Bedarf entspricht. Nur in der ambulanten Pflege können die Pflegekassen in diesem Fall eigene Pflegekräfte einstellen, in der stationären und insbesondere der wichtigen teilstationären Pflege gibt es keine entsprechende Vorschrift. Das Risiko einer zahlenmäßig unzureichenden ambulanten und teilstationären Versorgungssituation vor Ort tragen also vorrangig die Versicherten. ${ }^{47}$ Allerdings sind auch hier die Geschlechter unterschiedlich betroffen:

Männer werden zu Hause typischerweise durch ihre (gleichaltrigen oder jüngeren) Partnerinnen versorgt. Ist die pflegende Partnerin nicht (mehr) berufstätig, was häufig der Fall ist, kann bereits die ergänzende Versorgung durch einen ambulanten Pflegedienst ausreichend sein, um den Verbleib des pflegebedürftigen Mannes in der Häuslichkeit sicherzustellen. Für diesen Hauptfall der häuslichen Versorgung von Männern gibt es also in der Pflegeversicherung einen „backup “, denn sollten vor Ort nicht genügend ambulante Angebote vorhanden sein, könnte und müsste die Pflegeversicherung diese tatsächlich selbst sicherstellen.

Teilstationäre Angebote, die von der Pflegeversicherung nicht mit einem derartigen „back-up“ versehen sind, kommen demgegenüber eher Frauen zugute: Zum einen in ihrer Rolle als Pflegende, sei es, weil sie erwerbstätig sind (bei den nicht selten jüngeren Partnerinnen, die Hauptpflegeperson für Männer sind, und den ebenfalls noch recht häufig pflegenden Töchtern und Schwiegertöchtern ein nicht unwahrscheinlicher Fall), sei es, weil sie mit der Pflege zum Beispiel eines an Demenz erkrankten Mannes überfordert sind oder weil der (von der Pflegeversicherung nicht gedeckte) allgemeine Beaufsichtigungsbedarf mittlerweile 24 Stunden am Tag erreicht hat. Zum anderen sind teilstationäre Angebote gerade für Frauen eine Unterstützung, die selbst pflegebedürftig sind, insbesondere bei Demenzerkrankung. ${ }^{48}$ Denn diese werden - anders als Männer - ganz überwiegend von Töchtern oder Schwiegertöchtern gepflegt, welche sehr viel häufiger noch im erwerbsfähigen Alter sind als die Partnerinnen von pflegebedürftigen Männern. Daher ist eine häusliche Versorgung älterer Frauen häufig nur dann möglich, wenn teilstationäre Angebote die erwerbstätigen Pflegenden entsprechend entlasten. Fazit: Das Vorhandensein der für Frauen ungleich bedeutsameren teilstationären Angebote wird gesetzgeberisch ungleich weniger von der Pflegeversicherung unterstützt als die für die häusliche Versorgung von Männern relevanteren ambulanten Pflegeangebote. ${ }^{49}$

Faktisch wird das Problem der fehlenden ambulanten und teilstationären Angebote dann häufig dadurch gelöst - und dies führt auch dazu, dass der „Sicherstellungsauftrag“ der Pflegekassen bislang noch nie öffentlich durch die „Nachfra- ger" nach ambulanten oder teilstationären Diensten eingefordert wurde -, dass für die betroffenen Frauen die Versorgung in der stationären Pflegeeinrichtung, dem Pflegeheim, zur einzig möglichen Versorgungsform erklärt wird. Nach $\mathbb{} 43$ SGB XI besteht ein Anspruch auf stationäre Versorgung nämlich dann, wenn „häusliche oder teilstationäre Pflege nicht möglich“ ist. Da eine häusliche Versorgung in der Regel ein funktionierendes komplexes Pflegearrangement voraussetzt, kann das Fehlen ambulanter oder teilstationärer Angebote in der Region die häusliche/teilstationäre Pflege faktisch „unmöglich “ machen. Zwar ist dies vom Gesetzgeber eigentlich nicht gemeint, das Fehlen entsprechender Angebote führt aber nicht selten dazu, dass Pflegebedürftige und Angehörige ein häusliches Pflegearrangement als nicht realisierbar ansehen und wenn auch sicherlich mehrheitlich nicht gerne - die stationäre Versorgung als einzige realisierbare Alternative betrachten. Warum genau die häusliche oder teilstationäre Versorgung dann nicht möglich war, wird - insbesondere, wenn die Entscheidung über den Einzug in eine stationäre Pflegeeinrichtung schnell erfolgen musste - später nicht mehr gefragt. Ein „Rückumzug“ aus der Pflegeeinrichtung in die eigene Häuslichkeit oder den Haushalt von Angehörigen kommt praktisch nicht vor. Der oft diskutierte „Heimsog“ der Pflegeversicherung trifft also vorrangig Frauen.

Die Vorstellung von einem „Pflegemarkt“, wie ihn das Gesetz vorsieht, funktioniert also dort nicht, wo eine potentielle Nachfrage angesichts der Unkenntnis über die Möglichkeiten erst gar nicht entsteht. Auf diese Weise entsteht jedoch auch kein politischer Druck, der den Gesetzgeber dazu veranlassen könnte, hinreichende Anreize für den Ausbau teilstationärer Angebote zu setzen. Auch hier zeigt sich das SGB XI anbieter-, nicht nachfrageorientiert - ein Manko, das ebenfalls zur unzureichenden Ausprägung der Versorgungsinfrastruktur beiträgt. Zu lösen wäre diese Problematik durch die „Schaffung einer gesellschaftlichen Infrastruktur für Kinderbetreuung und Pflege, die bislang unbezahlt in Haushalten erledigt wurde" ${ }^{50}$; konkret: den konsequenten und am tatsächlichen Bedarf orientierten Ausbau von ambulanten und insbesondere teilstationären Angeboten, der nur durch eine deutliche Aufstockung der Finanzierung dieser Angebote und gegebenenfalls eine ergänzende Klarstellung des diesbezüglichen Sicherstellungsauftrags möglich ist, sowie den Ausbau haushaltsnaher Dienstleistungen. ${ }^{51} \mathrm{Ob}$ ein sogenanntes „persönliches Budget" in der Pflege dies für die Mehrheit der Betroffenen befördern würde, kann angesichts der Erfahrungen aus der Behindertenhilfe bezweifelt werden. Dennoch wäre die Schaffung eines Rechtsanspruchs auf ein persönliches Budget in der

47 Udsching, Peter/Schütze, Bernd, SGB XI, $\$ 69$ Rn. 4.

48 Frauen weisen in allen Altersstufen höhere Prävalenzraten bei Demenz auf (RKI, Themenheft Pflege, 2004).

49 Umso bedeutsamer sind innovative und alternative Konzepte, wie sie z.B. Astrid Henriksen in diesem Heft beschreibt.

$50 \mathrm{Vgl}$. Gutachten zum 1. Gleichstellungsbericht, a.a.O., S. 218.

51 Vgl. hierzu auch den Beitrag von Christa Larsen und Sigrid Rand, Die unendliche Geschichte der informellen Beschäftigung in deutschen Privathaushalten? in diesem Heft. 
Pflege aus den vorgenannten Gründen ein weiterer wichtiger Baustein auf dem Weg zu mehr Geschlechtergerechtigkeit in den Pflegearrangements. Flankiert werden muss dies durch ein weiter verbessertes, integriertes Beratungsangebot im Vorfeld und bei Pflegebedürftigkeit, das mit einer Unterstützung bei der Planung und Inanspruchnahme von Leistungen im Rahmen eines Pflegearrangements gekoppelt ist. Die bisherigen Regelungen der $\mathbb{S} \mathbb{S}$ und 7a SGB XI gehen zwar in die richtige Richtung. Sie bleiben jedoch in der Umsetzung hinter den Erfordernissen zurück, insbesondere fehlt es an der Koordination mit Beratungs- und Unterstützungsangeboten anderer Leistungsträger und der Kommunen (hier insbesondere im Rahmen der Altenhilfe des $\$ 71$ SGB XII, der aufgrund seiner präventiven und teilhabeorientierten Ausrichtung als relativ modern gelten kann und ein großes Entwicklungspotential im Kontext geschlechtergerechter Pflege hat).

In den Kontext der häuslichen Versorgung fällt auch die öffentlich viel diskutierte Problematik einer häuslichen Rundum-die-Uhr-Betreuung und -Versorgung. Hier ist zunächst klarzustellen, dass es sich nicht um eine 24-Stunden-Pflege handelt, wie sie für die Pflegestufe III beschrieben ist $(\mathbb{S} 15 \mathrm{Abs}$. 1 S. 1 Ziff. 3 SGB XI) und leistungsrechtlich abgesichert ist. Es geht vielmehr vorrangig um einen allgemeinen Aufsichts- und Betreuungsbedarf, wie er zum Beispiel bei Menschen mit demenziellen Veränderungen insbesondere im fortgeschrittenen Stadium der Krankheit (z.B. aufgrund von Weglauftendenzen, Störungen des Tag-Nacht-Rhythmus) bestehen kann. Dieser Bedarf wird von der Pflegeversicherung nicht und von der im Bedarfsfall ergänzend zu leistenden Hilfe zur Pflege des SGB XII (Sozialhilfe), hier insbesondere $\mathbb{} 65$ Absatz 1 Satz 2 SGB XII („angemessene Kosten für eine besondere Pflegekraft“), nur eingeschränkt erfasst. Zudem ist bei Sozialhilfeleistungen vorrangig das eigene Einkommen und Vermögen einzusetzen und bei unverhältnismäßigen Kosten ein Verweis auf eine stationäre Versorgung möglich, was den Kreis der Leistungsberechtigten (und Antragsteller(innen)) deutlich verkleinert.

Da die 24-Stunden-Betreuung leistungsrechtlich nicht abgesichert ist, die private Finanzierung einer Pflege- oder Haushaltshilfe jedoch mit durchschnittlich über 2.000 EUR pro Monat für viele kaum finanzierbar ist, wird teilweise auf (ausländerrechtlich sowie sozial- und steuerrechtlich illegal beschäftigte) ausländische, nicht selten osteuropäische Pflegeund Haushaltshilfen zurückgegriffen. ${ }^{52}$ Diese Tatsache wird zwar durch die Presse relativ überbetont, genaue Zahlen liegen jedoch naturgemäß nicht vor. Dennoch zeigt sich auch hier eine geschlechterspezifische Problematik, weil die illegal arbeitenden Frauen in sozial schlecht abgesicherten, (am Marktwert ihrer Arbeitsleistung gemessen) unterbezahlten Arbeitsverhältnissen ohne Arbeitsschutzvorschriften und politische Vertretung tätig sind. Allerdings ist diese Problematik vorwiegend eine Folge des in Deutschland politisch nicht konsequent umgesetzten Vorrangs der häuslichen Pflege und soll daher hier nicht vertieft behandelt werden. ${ }^{53}$

Ein Aspekt bezüglich der pflegerischen Versorgung von Frauen soll noch kurz erwähnt werden: Versorgung bedeutet nicht nur leistungsrechtliche Absicherung, sondern auch Sicherung der Qualität und Schutz der körperlichen und psychischen Unversehrtheit und Würde. Hier ist für die - überwiegend Frauen betreffende - stationäre Versorgung zunächst durch die kürzlichen Entwicklungen in der Qualitätssicherung des SGB XI und flankierende Forschungsprojekte ein Qualitätsschub für die stationäre Pflege zu erwarten. Die Qualitätssicherung in der häuslichen Pflege (diese erfolgt durch Beratungsbesuche von Pflegediensten) wird demgegenüber oft als unzureichend angesehen. Ein besonderes Problem hierbei stellt das Thema "Gewalt in der Pflege“ dar, das sowohl Pflegebedürftige als auch Pflegende betrifft. Hierzu soll auf den Bericht der kommunalen Beschwerdestelle München verwiesen werden, der gerade zur geschlechtsspezifischen Betroffenheit aufschlussreich ist. ${ }^{54}$

\section{Gleichstellungspolitische Analyse der Pflegepolitik für pfle- gende Angehörige und andere nicht professionelle Pflege- personen}

Das SGB XI würdigt die Leistungen Pflegender unmittelbar in den $\mathbb{S} \int 44 \mathrm{ff}$. SGB XI. In Bezug auf die soziale Sicherung gilt: Nach $\ 44$ Absatz 1 SGB XI zahlt die Pflegeversicherung für Pflegepersonen, d.h. informell Pflegende, die wenigstens 14 Stunden pro Woche pflegen ( $\mathbb{S} 19$ S. 2 SGB XI) und weniger als 30 Stunden pro Woche erwerbstätig sind ( $\mathbb{S} 44$ Abs. 1 S. 1 SGB XI) nach Pflegestufen und Zeitaufwand abgestufte Beiträge zur gesetzlichen Rentenversicherung $(\mathrm{GRV})^{55}$. Dies erfolgt durch eine Fiktion beitragspflichtiger Einnahmen der Pflegepersonen, nach deren Höhe die Beiträge anhand des jeweils geltenden Beitragssatzes bemessen werden. Je nach vorheriger Erwerbstätigkeit kann dies eine Schlechterstellung der Pflegenden in der gesetzlichen Rentenversicherung bedeuten.

Während der pflegerischen Tätigkeit sind sie nach $\mathbb{} 44 \mathrm{Ab}$ satz 1 Satz 6 SGB XI in den Versicherungsschutz der gesetzlichen Unfallversicherung einbezogen. Zudem können Pflegepersonen, die nach der Pflegetätigkeit in das Erwerbsleben zurückkehren wollen, bei beruflicher Weiterbildung nach Maßgabe des SGB III bei Vorliegen der dort genannten Voraussetzungen gefördert werden. Werden Pflegende aufgrund der Regelung des PflegeZG ${ }^{56}$ für bis zu sechs Monate vollständig von der Arbeitsleistung freigestellt oder wird ihre Beschäf-

52 Zum Themenkomplex insgesamt vgl. Scheiwe, Kirsten/Krawietz, Johanna (Hrsg.) (2010): Transnationale Sorgearbeit - Rechtliche Rahmenbedingungen und gesellschaftliche Praxis, VS Verlag für Sozialwissenschaften.

53 Zur pflegepolitischen Einordnung der sog. irregulären Beschäftigungsverhältnisse vgl. Hoffer, Heike (2010): Irreguläre Arbeitsmigration: Rechtliche und politische Argumente für das notwendige Ende einer politischen Grauzone, in: Scheiwe, Kirsten/Krawietz, Johanna, a.a.O., S. 95-120.

54 Vgl. den Beitrag von Kornelie Rahnema in diesem Heft.

55 Die entsprechenden Beiträge werden auf Antrag auch an berufsständische Versorgungseinrichtungen gezahlt, vgl. §44 Abs. 2 SGB $\mathrm{XI}$.

56 Das Pflegezeitgesetz gibt u.a. Beschäftigten in Betrieben mit in der Regel mehr als 15 Beschäftigten einen Rechtsanspruch auf bis zu 6 Monate unbezahlte vollständige oder teilweise Freistellung von der Arbeitsleistung, vgl. § 3 Abs. 1 PflegeZG. 
tigung durch die Reduzierung der Arbeitszeit zu einer geringfügigen Beschäftigung im Sinne des $\mathbb{8} 8$ Absatz 1 Nr. 1 SGB IV, erhalten sie nach $\mathbb{\int} 44 \mathrm{a}$ Absatz 1 SGB XI auf Antrag Zuschüsse zur Kranken- oder Pflegeversicherung, soweit sie nicht über eine beitragsfreie Familienversicherung versichert sind. Gewährt wird dabei die Höhe der Mindestbeiträge der freiwilligen oder gesetzlichen Kranken- und Pflegeversicherung, auch bei Privatversicherten. Pflegende sind darüber hinaus während der Inanspruchnahme der Pflegezeit arbeitslosenversichert. ${ }^{57}$

Das Pflegegeld nach $\$ 37$ SGB XI, das bei (mindestens auch) nicht professioneller Pflege an die Pflegebedürftigen zur Sicherstellung der Versorgung gezahlt wird, mag in vielen Fällen den informell Pflegenden (z.B. als faktische Einkommensaufstockung) zugutekommen, einen Rechtsanspruch hierauf haben sie aber nicht. Die vorgenannten Leistungen sind allerdings nur für Pflegepersonen, die eine(n) Pflegebedürftige(n) im Sinne der $\mathbb{S} \mathbb{S} 14,15$ SGB XI pflegen, verfügbar. Pflegende, die Menschen betreuen und versorgen, die nicht erheblich pflegebedürftig im Sinne des $\mathbb{} 14$ Absatz 1 SGB XI sind, aber dennoch einen hohen Bedarf an Beaufsichtigung und Betreuung haben (z.B. bei Demenzkranken aufgrund von kognitiven Beeinträchtigungen), werden von diesen Regelungen nicht erfasst.

Ergänzt werden die Leistungen zur sozialen Sicherung durch verschiedene Unterstützungsangebote für Pflegende: \45 SGB XI (Pflegekurse für Angehörige und sonstige an einer ehrenamtlichen Pflegetätigkeit interessierte Personen) und $\mathbb{S} 45 \mathrm{~b}$ SGB XI (Zusätzliche, qualitätsgesicherte Betreuungsleistungen im Gegenwert bis zu 200 EUR pro Monat insbesondere für die Entlastung von der häuslichen Betreuung kognitiv beeinträchtigter Menschen, z.B. Demenzkranken) sowie auf infrastruktureller Ebene im Rahmen der Weiterentwicklung der Versorgungsstrukturen der Ausbau von insbesondere niedrigschwelligen Betreuungsangeboten $(\mathbb{S} 45 \mathrm{c}$ Abs. 3 SGB XI) und der modellhaften Förderung von Möglichkeiten zur Vernetzung der Hilfen für Demenzkranke sowie der Förderung ehrenamtlicher Strukturen sowie der Selbsthilfe $(\mathbb{S} 45 \mathrm{~d}$ SGB XI).

Die genannten Leistungen für Pflegende sind jedoch aus gleichstellungspolitischer Sicht nicht ausreichend. Dabei ist die Bewertung zwiespältig: Betrachtet man allein die Situation von Frauen als Pflegenden, könnten höhere finanzielle Leistungen (erhöhtes Pflegegeld oder echte Lohnersatzleistungen bei Reduzierung von Erwerbsarbeit zugunsten von Pflegetätigkeiten) jedenfalls die ökonomische Situation Pflegender und gegebenenfalls auch beispielsweise die Absicherung im Alter verbessern. Allerdings können hierdurch (weitere) negative Erwerbsanreize für Frauen gesetzt werden, die die bestehenden Effekte verstärken können. ${ }^{58}$ Zwiespältig äußert sich auch der 1. Gleichstellungsbericht: So führten Erwerbsunterbrechungen - bei Frauen mit Kindern oder pflegebedürftigen Angehörigen verbreitet - trotz der geleisteten gesellschaftlich wichtigen Sorgearbeit zu erheblichen Nachteilen im weiteren Erwerbsleben. ${ }^{59}$ Hinzu kommt in der gesetzlichen Rentenversicherung eine deutliche Benachteiligung der Sorgearbeit für Pflegebedürftige im Vergleich zur Sorgearbeit für Kinder. ${ }^{60} \mathrm{Zu}$ fordern ist daher - auch an anderen Stellen im Sozialrecht eine Überprüfung und gegebenenfalls Anpassung der sozialrechtlichen Behandlung der Sorgearbeit von Pflegenden mit der Sorgearbeit für Kinder.

Nach wie vor ist der Wunsch nach familiärer Pflege bei Pflegebedürftigen wie Angehörigen groß. Damit dieser Wunsch nicht für Frauen zu geschlechtsspezifischen Benachteiligungen führt, ist eine bessere Vereinbarkeit von Pflegeund Erwerbstätigkeit erforderlich. Nach Feststellung der Gutachter(innen) wird eine sozialversicherungspflichtige Teilzeit von vielen Frauen (bisher selten von Männern) gewünscht und stellt sich für bestimmte Lebensphasen als geeignetes Vereinbarkeitsinstrument dar - insbesondere, wenn sie mit Rückkehroptionen auf eine Vollzeittätigkeit verbunden ist. ${ }^{61}$ Der nun vorliegende Regierungsentwurf für eine Familienpflegezeit $^{62}$ ist nach der Bewertung der djb-Kommission Recht der sozialen Sicherung, Familienlastenausgleich jedoch hierfür nur unzureichend geeignet und begegnet gleichstellungspolitischen Bedenken: Denn zum einen sichert er mit einer nur zweijährigen, recht starren Familienpflegezeit die Pflegedauern nur teilweise ab, zum anderen dient er mit seiner „Ausfallversicherung “ zugunsten des Arbeitgebers, wenn (die) der Arbeitnehmer(in) das während der Pflegezeit aufgestockte Wertguthaben nicht zurückzahlen kann, vorrangig als „Arbeitgeberschutzgesetz“, nicht als Schutzgesetz zugunsten der Pflegenden. Von zentraler Bedeutung ist demgegenüber der Rückkehranspruch auf die vorherige Arbeitszeit - dieser hätte jedoch auch über bestehende gesetzliche Regelungen des Teilzeit- und Befristungsgesetzes gelöst werden können. Ergänzend fordern die Gutachter(innen) zum 1. Gleichstellungsbericht, dass die Betriebe auf die Bedürfnisse von Pflegenden stärker als bisher im Rahmen einer lebensereignisorientierten Personalpolitik eingehen müssen. ${ }^{63}$

Schließlich stehen die gesundheitlichen und sozialen Teilhaberisiken Pflegender bislang kaum im Fokus der politischen Überlegungen und werden in den $\mathbb{S} \mathbb{S} 44 \mathrm{ff}$. SGB XI nur insoweit berücksichtigt, als zum Beispiel Selbsthilfegruppen Pflegender gefördert werden. Einzelleistungsansprüche existieren kaum. Dementsprechend fordern auch die Gutachter(innen) zum 1. Gleichstellungsbericht, dass ein geschlechtergerechtes Sozialrecht vor dem Hintergrund der gesetzlich und von der Mehrheit der Betroffenen bevorzugten häuslichen Pflege und der hiermit verbundenen physischen und psychischen Belastungen die Leistungsansprüche der überwiegend weiblichen Pflegepersonen so ausgestalten muss, dass Teilhaberisiken für

57 Hierauf weist § 44a Abs. 2 SGB XI, der aber nicht selbst anspruchsbegründend wirkt, sondern auf die entsprechenden Vorschriften des SGB III verweist, vgl. Klie, Thomas/Krahmer, Utz/Schiffer-Werneburg, Marie-Luise, § 44a, Rn. 13.

58 Vgl. Rothgang, Heinz et al., a.a.O., S. 87.

59 Vgl. Gutachten zum 1. Gleichstellungsbericht, a.a.O., S. 213.

60 Vgl. Gutachten zum 1. Gleichstellungsbericht, a.a.O., S. 216.

61 Vgl. Gutachten zum 1. Gleichstellungsbericht, a.a.O., S. 213.

62 Der Gesetzentwurf liegt mittlerweile - in leicht veränderter Fassung - als Regierungsentwurf dem Bundesrat als Drucksache 20711 vom 15.4.2011 vor (Stand: 28.4.2011).

63 Vgl. Gutachten zum 1. Gleichstellungsbericht, a.a.O., S. 221. 
die Pflegenden möglichst vermieden oder mindestens so weit wie möglich abgemildert werden. ${ }^{64}$ Schließlich dient auch eine bessere Versorgungsinfrastruktur, wie sie bereits oben unter dem Kapitel über Pflegebedürftige beschrieben wurde, ergänzt durch ausgebaute Leistungen der Verhinderungs- und Kurzzeitpflege ( $\mathbb{S}$ 39, 42 SGB XI) den Bedürfnissen Pflegender.

\section{Gleichstellungspolitische Analyse der Pflegepolitik für pro- fessionelle Pflegekräfte}

Auch in der professionellen Pflege gibt es eine gleichstellungspolitische Problematik. ${ }^{65}$ Charakterisiert wird der „Frauenjob“ der Pflege durch eine vergleichsweise schlechte Bezahlung für eine stark belastende Tätigkeit. ${ }^{66}$ Zudem sind Pflegeberufe wie viele Dienstleistungsberufe immer noch als Helferinnen-, Assistentinnen- und Zuverdienerinnen-Stellen mit schlechten Weiterbildungs- und Aufstiegsmöglichkeiten konzipiert. ${ }^{67}$ Die vollzeitschulische Ausbildung zementiert mit uneinheitlichen Qualifikationsprofilen und fehlenden bundesweiten Standardisierungen den geringeren Professionalisierungsgrad vieler typischer Frauenberufe wie der Pflege. ${ }^{68}$ Die Arbeitsbedingungen (Schichtarbeit, körperliche und psychische Belastungen) führen häufig zu Teilzeitarbeit - wenn diese möglich ist - und frühem Berufsausstieg bzw. einem fehlendem Wiedereinstieg nach einer Familienphase. ${ }^{69}$ Zudem einen findet eine Gender-Hierarchisierung innerhalb der professionellen Pflege statt („Männer im Management, Frauen am Bett“"). ${ }^{70}$

Politisch wurde in dieser Legislaturperiode durch die Einführung eines gesetzlichen Mindestlohns (8,50 Euro in Westdeutschland, 7,50 Euro in Ostdeutschland) reagiert. Weitere politische Initiativen reagieren jedoch vorrangig auf die Problematik eines (drohenden) Fachkräftemangels in der Pflege und weniger auf die gleichstellungspolitische Problematik (z.B. durch Qualifizierungs- und Akademisierungsmöglichkeiten und Schaffung einer integrierten Pflegeausbildung), auch wenn diese sich teilweise gleichstellungspolitisch positiv auswirken könnten.

$\mathrm{Zu}$ beobachten ist aus gleichstellungspolitischer Sicht auch die oft geäußerte Forderung nach weiterer Ausdifferenzierung der Aufgabenprofile in der Pflege, die zu einer Abschichtung der Aufgaben und Ausdifferenzierung (faktisch: Absenkung) der Entlohnung für bestimmte Aufgabenanteile führen soll (vgl. z.B. die zusätzlichen Betreuungskräfte nach $\$ 87 \mathrm{~b}$ SGB XI). Diese Entwicklung hat zwei Facetten: Einerseits kann sie für die „semiprofessionell“ Beschäftigten zu neuen, letztlich prekären Beschäftigungsformen führen, die dann wieder überwiegend von Frauen in Anspruch genommen werden. Zum anderen werden Pflegearrangements hierdurch möglicherweise finanziell auch bei legaler Beschäftigung erschwinglicher für die weiblichen Pflegebedürftigen ein Vorteil.

\section{Forschungsbedarfe und politische Handlungsmöglichkeiten}

Die Feststellung, dass Pflege weiblich ist, findet - dem entspricht die hier vorgenommene Darstellung - in der Literatur noch überwiegend anhand von quantitativen Betrachtungs- weisen statt. Diese können zwar auch für sich genommen bestimmte Annahmen und Schlussfolgerungen implizieren. Wünschenswert wäre jedoch die Herstellung von Begründungszusammenhängen, insbesondere in der Lebenslaufperspektive, die die Analyse und Handlungsempfehlungen zu einer Veränderung weiter unterstützen könnten. Auch die Betrachtung von qualitativen Aspekten unterbleibt weitgehend. ${ }^{71}$ Selbst die Gründe für die geschlechtsspezifisch höhere Lebenserwartung und das größere Pflegerisiko von Frauen sind nur teilweise bekannt. ${ }^{72}$

Nichtsdestotrotz bestehen auch zum gegenwärtigen Zeitpunkt politische Handlungsbedarfe und -möglichkeiten. Denn auch wenn die genannten Aspekte Frauen überproportional häufig treffen und es diesbezügliche Forschungsbedarfe gibt, treffen die Probleme alle Beitragszahler(innen), Pflegebedürftigen, privat und professionell Pflegenden. Hier sind schon jetzt deutliche Verbesserungen nötig und möglich. Zum einen ist wenn der Vorrang der häuslichen Pflege als Grundsatz der Pflegeversicherung bestehen bleiben soll - die Pflegeversicherung auch entsprechend dieses Vorrangs auszugestalten. Dies bedeutet, dass die häusliche Pflege eine erheblich stärkere Unterstützung erfahren muss als bisher - auch zulasten des stationären Sektors. Zum anderen sind die Vermeidung und Verringerung von Pflegebedürftigkeit und flankierende Unterstützungsbedarfe erheblich auszubauen.

Der in dieser Legislaturperiode umzusetzende neue Pflegebedürftigkeitsbegriff bietet einige grundlegende Potentiale zu einer Umsteuerung der Pflegepolitik, eine Neuausrichtung der Leistungen und eine Neugestaltung des Verhältnisses zwischen Pflegeversicherung und dem SGB XII-Leistungsträger. ${ }^{73}$ Der mögliche Zugewinn für Frauen aus einer solchen Reform ist erheblich. Daher tut der djb gut daran, sich in diesen Prozess aktiv einzubringen und ihn kritisch und konstruktiv zu begleiten. Schritte in diese Richtung wurden in den vergangenen Jahren in der Arbeit verschiedener Kommissionen und Arbeitsgruppen unternommen und werden gegenwärtig in der AG Pflege/Fachgruppe Vorsorge der Kommission Recht der sozialen Sicherung, Familienlastenausgleich ${ }^{74}$ fortgesetzt. Dieser und die nachfolgenden Beiträge sollen weitere Handlungsfelder aufzeigen.

64 Vgl. Gutachten zum 1. Gleichstellungsbericht, a.a.O., S. 221.

65 Diese wird z.B. von Backes, Gertrud et al. (2008), a.a.O., S. 48 ff. ausführlich beschrieben.

66 Backes, Gertrud et al. (2008), a.a.O., S. $48 \mathrm{ff}$

67 Vgl. Gutachten zum 1. Gleichstellungsbericht, a.a.O., S. 212, 213.

68 Vgl. Gutachten zum 1. Gleichstellungsbericht, a.a.O., S. 212.

69 Backes, Gertrud et al. (2008), a.a.O., S. $48 \mathrm{ff}$.

70 Backes, Gertrud et al. (2008), a.a.O., S. $48 \mathrm{ff}$

71 Vgl. auch Backes, Gertrud et al. (2008), a.a.O., S. 136.

72 Robert Koch Institut (2004), a.a.O., S. 15.

73 Siehe hierzu auch: Hoffer, Heike/Rasch, Edna (2010): Ein neuer Pflegebedürftigkeitsbegriff - Herausforderungen an die Umsetzung, in: Archiv für Wissenschaft und Praxis der sozialen Arbeit, Heft 3, S. 4-13.

74 Ansprechpartnerin für die Kommission Recht der sozialen Sicherung, Familienlastenausgleich ist Prof. Dr. Margarete SchulerHarms; für die Fachgruppe „Vorsorge“ Prof. Dr. Astrid Wallrabenstein; für die AG Pflege Ass. iur. Heike Hoffer, LL.M. (Vanderbilt). 